\title{
Face Recognition based Attendance System
}

\author{
Dhanush Gowda H.L ${ }^{1}$, K Vishal ${ }^{2}$, Keertiraj B. R ${ }^{3}$, Neha Kumari Dubey ${ }^{4}$, Pooja M. R. ${ }^{5}$ \\ ${ }^{1,2,3,4}$ Department of Computer Science and Engineering \\ ${ }^{5}$ Associate Professor, Department Science and Engineering Vidyavardhaka College of Engineering, Mysuru, Karnataka, India
}

\begin{abstract}
The management of the attendance can be a great burden on the teachers if it is done by hand. To resolve this problem, smart and auto attendance management system is being utilized. By utilizing this framework, the problem of proxies and students being marked present even though they are not physically present can easily be solved. This system marks the attendance using live video stream. The frames are extracted from video using OpenCV. The main implementation steps used in this type of system are face detection and recognizing the detected face, for which dlib is used. After these, the connection of recognized faces ought to be conceivable by comparing with the database containing student's faces. This model will be a successful technique to manage the attendance of students.
\end{abstract}

Keywords: Attendance system, Automated attendance, Image Processing, Face detection, Feature matching, Face recognition.

\section{I.INTRODUCTION}

Human face plays an important role in our day to day life mostly for identification of a person. Face recognition is a part of biometric identification that extracts the facial features of a face, and then stores it as a unique face print to uniquely recognize a person. Biometric face recognition technology has gained the attention of many researchers because of its wide application. Face recognition technology is better than other biometric based recognition techniques like finger-print, palm-print, iris because of its non-contact process. Recognition techniques using face recognition can also recognize a person from a distance, without any contact or interaction with person. The face recognition techniques are currently implemented in social media websites like Facebook, at the airports, railway stations. The, at crime investigations. Face recognition technique can also be used in crime reports, the captured photo can be stored in a database, and can be used to identify a person. Facebook uses the facial recognition technique for automating the process of tagging people. For face recognition we require large dataset and complex features to identify a person in all conditions like change of illumination, age, pose, etc. Recent researches show there is a betterment in facial recognition systems. In the last ten years there is huge development in recognition techniques.

But currently most of the facial recognition techniques is able to work fine only if the number of people in one frame is very few and under controlled illumination, proper position of faces and clear images. For face recognition purpose, there is a need for large data sets and complex features to uniquely identify the different subjects by manipulating different obstacles like illumination, pose and aging. During the recent few years, a good improvement has been made in facial recognition systems. In comparison to the last decade, one can observe an enormous development in the world of face recognition. Currently, most of the facial recognition systems perform well with limited faces in the frame. Moreover, these methodologies have been tested under controlled lighting conditions, proper face poses and non- blurry images. The system that is proposed for face recognition in this paper for attendance system is able to recognize multiple faces in a frame without any control on illumination, position of face.

\section{II.RELATED WORKS}

The paper "Individual Stable Space: An Approach to Face Recognition Under Uncontrolled Conditions" by Xin Geng, says that most face recognition systems needs the faces to be fed into it based on certain rules, like under a controlled illumination, at a particular position, under a particular view angle, and without any obstacles. Such systems are called face recognition under controlled conditions. These rules restrict the uses of face recognition in many real time applications because they cannot satisfy these rules. Real time applications need techniques which does not need any strict control over the human beings for recognizing the face. These types of systems need face recognition under uncontrolled conditions. So, this paper proposes one such system but the system needs an image as input and one person per image which is a drawback of the system and provides a hindrance in using it in real time applications like attendance systems.[1]

In the paper "Anti-Cheating Presence System Based on 3WPCA-Dual Vision Face Recognition”, Edy Winarno proposed a system that can predict the cheating in facial recognition-based system like using the photograph of an authorized person or image similar to the authorized person. They used dual vision camera also called as stereo vision camera which produces one image from each of its two lenses. After getting the two images they used half-join method to combine the half of left image and half of right image of a person into a single image of the person that can then undergo extraction using 3WPCA method. The recognition of cheating using this system is $98 \%$.[2] In this paper the author designed and explained improvement of picture-based attendance system capture 
the faces of many students and may be the next generation to all the bio-metric devices that are ruling now. Human face, a differential thing and has a great degree of changing tendency, so it needs to be fast and accurate for detecting student's facial structures. Processing the system will involve registration of students by taking their images and then taking them for setting Attendance. Continuous registration is required to achieve great and sharp accuracy. In this system. This paper tells the system and lastly evidence will be given to support the system. The project can be used in online examination for certification. Identification of the student taking the test. [3].

There are a number of systems for attendance purpose, like traditional methods of data, have drawbacks and hard to use that list, a biometric presence. There is a lack of human error in the system like fingerprint scan is not accepted because of wet conditions Fingers, dirty, very dry or peeled fingers. So, the author proposes Authority to add mobile presence system and face with NFC Safety facility and possibility to store using Raspberry Pi data in the cloud. The paper reviews relatable works. Attendance management system, NFC, face authority area, Microcomputers and Cloud area. Then, it provides new Method and design system and planning. Outcome of this is a system which reduces the usage of paper, ending time and energy wasted by attendance Mobile Based Attendance System.[4]

Computers are intelligent to communicate with humans in various perspectives. It will be participation If it is based then it is more acceptable for both humans and computers. On the validation process. The author is concerned Integrating and developing a student recognition using "survival- Ing" algorithm. Then embedding is used in classification of a person's face the system offers a variety of applications such as attendance - Systems, security etc. After making a system, a resulting display, is shown in the paper.[5]
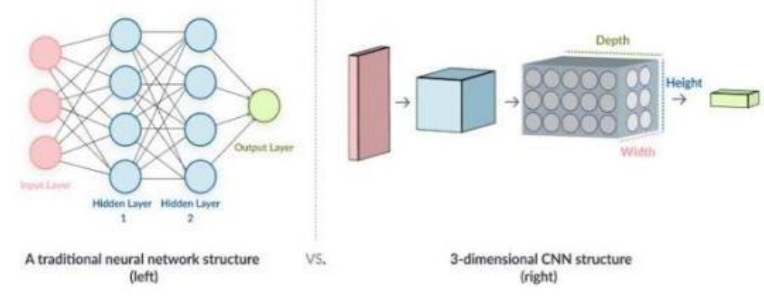

Figure 1: Traditional neural network versus CNN.

In this paper "Face Recognition based Attendance System using Machine Learning Algorithms" by Radhika C. Damale, the author says identification of a person by facial features Known as facial recognition. A face feature can be used for various computer-based vision algorithms such as face recognition, emotion detection and multiple camera surveillance applications. Face recognition system is attracting scholars towards it. In this, different methods such as SVM, MLP and CNN are discussed. DNN is used to "face detection". For SVM and MLP approaches, the features like PCA and LDA extracted using extraction algorithms. In CNN approach, images fed directly to CNN
Module as a feature. The approach shows Good detection accuracy percentage for CNN based approaches. SVM, MLP and CNN achieve test accuracy of $87 \%, 86.5 \%$ and $98 \%$ on self- generated databases respectively. [6]

In the paper" Class Attendance framework the on- Face Recognition" composed by Priyanka Wagh. To distinguish the understudies sitting on the last columns conveniently, the histogram leveling of picture should be finished. The picture will be passed for individual's face discovery. The productivity of Ada-Boost calculation is best of all these. In this way, this will paper utilizes this calculation for identifying countenances of understudies by utilizing the haar highlight classifiers and course ideas of Ada-Boost calculation .Every understudy's face is trimmed and the different highlights are removed from them like separation between eyes, nose, blueprint of face, and so forth utilizing these countenances as Eigen includes, the understudy is perceived and by contrasting them and the face database and their participation are stamped. A database of faces should be made with the end goal of examination. [7]

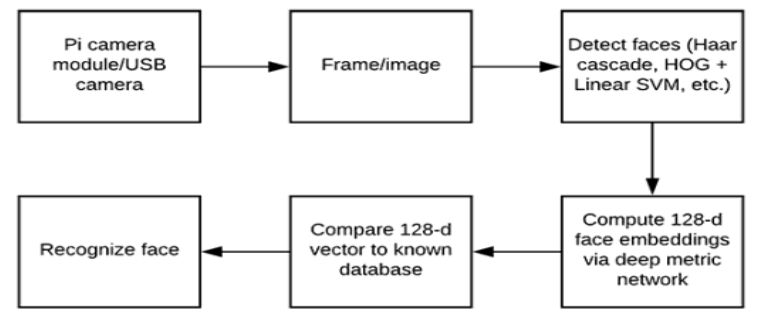

Figure 2: Face Recognition model

The classroom attendance system based on face recognition technology uses the camera to monitor the scene information. It triggers the shooting of the student face photo event, reads the student information when the student is signed in with campus card, which prevents nonschool personnel from entering the classroom and substitute classes. [8]

In the paper" Computerized Participation Framework Utilizing Face Acknowledgment "composed by Akshara Jadhav, Akshay Jadhav Tushar Ladhe, Krishna Yeolekar. the recognized face is separated and exposed to prepreparing. This pre-preparing step includes with histogram leveling of the extricated face picture and is resized to $100 \times 100$. In this framework, in the wake of perceiving the essences of the understudies, the names are refreshed into an exceed expectations sheet. The exceed expectations sheet is created by trading instrument present in the database framework. The database likewise can create month to month and week after week reports of understudy's participation records. catch the Understudy's Picture Apply Face identification calculations to recognize face extricate the locale of enthusiasm for rectangular bouncing box convert to dim scale, apply histogram adjustment and resize to $100 \times 100$ for example apply prepreparing on the off chance that enrolment stage, at that point stores in database else apply PCA/LDA/LBPH (for highlight extraction) [9].

In the paper "An Attendance Marking System based on Face Recognition" written by Khem Puthea, Rudy Hartanto and Risanuri Hidayat, says that the proposed system uses a 
machine learning technique named as principal component analysis or PCA for face recognition and other machine learning algorithms used in computer vision. A technique called Haar classifier is used to train the system to detect a face. When the faces are captured by a camera, they are first converted to grayscale and then to that image subtraction process is applied. The image after this is stored on the server for further processing which is done later. [10]

The author proposed a strategy where the framework was used as an online Web Server, so the participation results can be open to a verified web customer. The facial acknowledgment is finished by actualizing Local Binary Patterns (LBP) first handling venture is to identify and edit the locale of intrigue ROI which is the human face, then apply the Haar Feature-based Cascade calculation After that, the picture highlights are extricated utilizing LBPs, at that point LBPs calculation contrasts the separated highlights and the prepared datasets. Later, by clicking ' $c$ ' as in catch on the console framework, the participation results are put away in MySQL database, so it tends to be available to the web server. [11]

In the paper "Face Recognition Based Attendance System "written by Nandhini R. the author mentions the fundamental working rule of the venture is that, the video caught information is changed over into picture to identify and perceive it. CNN calculation is executed to recognize the faces. A CNN (Convolution Neural Network), utilizes a framework like a multilayer perceptron, intended to process the prerequisites faster. After the end of distinguishing and preparing the face, it is contrasted with the face's present in the understudy's database to refresh the participation of the students. The post- preparing component includes the way toward refreshing the names of the understudy into an exceed expectations sheet. The exceed expectations sheets can be kept up on a week after week premise or month to month premise to record the understudy's participation.[12].
In the paper "Student Attendance System in Classroom Using Face Recognition Technique "composed by Samuel Lukas, Aditya Rama Mitra, Ririn Ikana Desanti, Dion Krisnadi" the author says the quantity of highlights of any facial understudy picture is made to be consistent, for example 16 DCT coefficient. The process is finished entirely performing grayscale standardization, histogram balance, Discrete Wavelet Transform (DWT), and Discrete Cosine Transform (DCT). Further examination of the disappointment in perceivingthe rest facial pictures demonstrates that an understudy might be perhaps perceived as other student(s)By considering the all-out degree of acknowledgment as came about because of the investigation which doesn't meet exclusive requirement.[13]

In the paper "Attendance System based on Face Recognition" written by Venkata Kalyan Polamarasetty, Muralidhar Reddy Reddem, Dheeraj Ravi, Mahith Sai Madala.they proposed that we need to catch the picture from the webcam or the outside camera.] To do as such, in MATLAB, they introduce the drivers from the math works site dependent on the sort of camera we are utilizing.

Next, they use any rate 500 to 1000 catches of every individual. For getting higher level of exactness they used some HD camera so as to get results. For face distinguishing, we can do it utilizing the article falling class and we utilize the b-box technique. The caught countenances are trimmed into little pictures of goals $112 \times 92$. It would associate with $11 \mathrm{~KB}$ of size. The faces taken in the database are expected to stack into our workspace. We will stack the gallery images into that. All the HOG highlights separated are put away as exhibit list it restores a mark to which the given information matches or about coordinated.[14] 
Table 1: Comparison of different techniques used in face recognition-based attendance system.

\begin{tabular}{|c|c|c|c|c|}
\hline $\begin{array}{l}\text { Ref } \\
\text { No. }\end{array}$ & $\begin{array}{l}\text { Title \& } \\
\text { Authors Name }\end{array}$ & Concept Used & Advantages & Disadvantages \\
\hline 1. & $\begin{array}{l}\text { Individual } \\
\text { Stable Space: } \\
\text { An Approach } \\
\text { to Face } \\
\text { Recognition } \\
\text { Under } \\
\text { Uncontrolled } \\
\text { Conditions Xin } \\
\text { Geng, Zhi-Hua } \\
\text { Zhou, \& } \\
\text { Smith-Miles }\end{array}$ & $\begin{array}{l}\text { Face } \\
\text { recognition } \\
\text { under } \\
\text { uncontrolled } \\
\text { condition. [1] }\end{array}$ & $\begin{array}{l}\text { This paper } \\
\text { projects on } \\
\text { face } \\
\text { recognition } \\
\text { under } \\
\text { uncontrolled } \\
\text { conditions. }\end{array}$ & $\begin{array}{l}\text { Video } \\
\text { sequences, } \\
\text { verification, } \\
\text { and multiple } \\
\text { persons per } \\
\text { image } \\
\text { required by } \\
\text { most of the } \\
\text { real } \\
\text { applications } \\
\text { can't be } \\
\text { implemented. }\end{array}$ \\
\hline 2. & $\begin{array}{l}\text { Anti-Cheating } \\
\text { Presence } \\
\text { System Based } \\
\text { on 3WPCA } \\
\text { Dual Vision } \\
\text { Face } \\
\text { Recognition } \\
\text { Winarno, } \\
\text { Wiwien } \\
\text { Hadikurniawat } \\
\text { i, Imam Husni } \\
\text { Al Amin, Muji } \\
\text { Sukur }\end{array}$ & $\begin{array}{l}\text { Dual vision } \\
\text { face } \\
\text { recognition } \\
\text { using } \\
\text { 3WPCA. [2] }\end{array}$ & $\begin{array}{l}\text { It can } \\
\text { anticipate } \\
\text { falsification } \\
\text { of face data } \\
\quad \text { with } \\
\text { recognition } \\
\text { accuracy up to } \\
98 \%\end{array}$ & $\begin{array}{l}\text { The relative } \\
\text { angle of the } \\
\text { target's face } \\
\text { influences } \\
\text { the } \\
\text { recognition } \\
\text { score } \\
\text { profoundly. }\end{array}$ \\
\hline 3. & $\begin{array}{l}\text { Prototype } \\
\text { model for an } \\
\text { Intelligent } \\
\text { Attendance } \\
\text { System based } \\
\text { on facial } \\
\text { IdentificationR } \\
\text { aj Malik, } \\
\text { Praveen } \\
\text { Kumar, Amit } \\
\text { Verma, Seema } \\
\text { Rawat }\end{array}$ & $\begin{array}{l}\text { ADA Boost } \\
\text { algorithm with } \\
\text { techniques } \\
\text { PCA and LDA } \\
\text { Hybrid } \\
\text { algorithm [7]. }\end{array}$ & $\begin{array}{l}\text { By using this } \\
\text { system } \\
\text { chances of } \\
\text { fake } \\
\text { attendance } \\
\text { and proxy can } \\
\text { be reduced. }\end{array}$ & $\begin{array}{l}\text { Works only } \\
\text { for single } \\
\text { image of a } \\
\text { system. }\end{array}$ \\
\hline 4. & $\begin{array}{l}\text { Convolutional } \\
\text { Neural } \\
\text { Network } \\
\text { Nusrat Mubin } \\
\text { Ara1. Neural } \\
\text { Network } \\
\text { Approach for } \\
\text { Vision Based } \\
\text { Student } \\
\text { Recognition } \\
\text { System }\end{array}$ & $\begin{array}{lr}\text { Alex } & \text { NET } \\
\text { CNNs } & \text { and } \\
\text { RFID } & \\
\text { Technology } \\
{[8] .}\end{array}$ & $\begin{array}{l}\text { Uses the } \\
\text { camera system } \\
\text { to Monitor the } \\
\text { scene } \\
\text { information. }\end{array}$ & $\begin{array}{l}\text { Alex NET } \\
\text { won't work on } \\
\text { all the students } \\
\text { until it is } \\
\text { improved. } \\
\text { RFID } \\
\text { technology } \\
\text { uses electronic } \\
\text { toy which can't } \\
\text { be used in all } \\
\text { the cases. }\end{array}$ \\
\hline 5. & $\begin{array}{l}\text { NFC Based } \\
\text { Mobile } \\
\text { Attendance } \\
\text { System with } \\
\text { Facial } \\
\text { Authorization } \\
\text { on Raspberry } \\
\mathrm{Pi} \text { and Cloud } \\
\text { Server Siti } \\
\text { Ummi } \\
\text { Masruroh } \\
\end{array}$ & $\begin{array}{l}\text { Iris } \\
\text { Recognition } \\
{[9] .}\end{array}$ & $\begin{array}{l}\text { Real time face } \\
\text { detection and } \\
\text { efficient }\end{array}$ & \begin{tabular}{lr}
\multicolumn{3}{l}{ Iris condition } \\
needs to \\
improve in \\
different \\
light conditions
\end{tabular} \\
\hline 6. & $\begin{array}{l}\text { Facerecognitio } \\
\text { n-based } \\
\text { Attendance } \\
\text { System using } \\
\text { MachineLearni } \\
\text { ng Algorithms } \\
\text { Radhika C. } \\
\text { Damale }\end{array}$ & $\begin{array}{l}\text { Local Binary } \\
\text { patterns } \\
\text { (Support } \\
\text { vector } \\
\text { machine), } \\
\text { LDA based } \\
\text { OpenCV and } \\
\text { FLTK.[10]. }\end{array}$ & $\begin{array}{l}\text { Continuous } \\
\text { and automatic } \\
\text { attendance } \\
\text { system. }\end{array}$ & $\begin{array}{l}\text { System has } \\
\text { issues with } \\
\text { system } \\
\text { performance } \\
\text { and accuracy. }\end{array}$ \\
\hline 7. & $\begin{array}{l}\text { ClassAttendanc } \\
\text { esystem based } \\
\text { on Face } \\
\text { Recognition" } \\
\text { Priyanka } \\
\text { Wagh. }\end{array}$ & $\begin{array}{l}\text { Local Binary } \\
\text { patterns } \\
\text { (Support } \\
\text { vector } \\
\text { machine), } \\
\text { LDA } \\
\text { based } \\
\text { OpenCV and } \\
\text { FLTK.[11]. }\end{array}$ & $\begin{array}{l}\text { continuous } \\
\text { and automatic } \\
\text { attendance } \\
\text { system. }\end{array}$ & $\begin{array}{l}\text { System has } \\
\text { issues with } \\
\text { system } \\
\text { performance } \\
\text { and accuracy. }\end{array}$ \\
\hline
\end{tabular}

\begin{tabular}{|c|c|c|c|c|}
\hline 8. & $\begin{array}{l}\text { Design of } \\
\text { Classroom } \\
\text { Attendance } \\
\text { System Based } \\
\text { on Face } \\
\text { Recognition, } \\
\text { Wenxian Zeng }\end{array}$ & $\begin{array}{l}\text { CNN } \\
\text { uses a system } \\
\text { like } \\
\text { multilayer a } \\
\text { perceptron } \\
\text { that has been } \\
\text { designed to } \\
\text { process the } \\
\text { requirements } \\
\text { faster.[12] }\end{array}$ & $\begin{array}{l}\text { The Automated } \\
\text { Classroom } \\
\text { Attendance } \\
\text { System helps in } \\
\text { increasing the } \\
\text { accuracy and } \\
\text { speed ultimately } \\
\text { achieve the } \\
\text { high- precision } \\
\text { real-time } \\
\text { attendance to } \\
\text { meet the need } \\
\text { for automatic } \\
\text { classroom } \\
\text { evaluation. }\end{array}$ & $\begin{array}{l}\text { Other methods } \\
\text { with greater } \\
\text { accuracy can be } \\
\text { used to build }\end{array}$ \\
\hline 9. & $\begin{array}{l}\text { Automated } \\
\text { Attendance } \\
\text { System Using } \\
\text { Face } \\
\text { Recognition } \\
\text { Akshara Jadhav, } \\
\text { Akshay Jadhav } \\
\text { Tushar Ladhe, } \\
\text { Krishna } \\
\text { Yeolekar }\end{array}$ & DWT & $\begin{array}{l}\text { Facial images } \\
\text { can be } \\
\text { recognized } \\
\text { successfully } \\
\text { giving a total } \\
\text { level of } \\
\text { recognition of } \\
82 \% \text {. This figure } \\
\text { is perceived as } \\
\text { the best } \\
\text { recognition rate } \\
\text { which can be } \\
\text { obtained from } \\
\text { the data. }\end{array}$ & $\begin{array}{l}\text { Higher accuracy } \\
\text { can be obtained. }\end{array}$ \\
\hline 10. & $\begin{array}{l}\text { An Attendance } \\
\text { Marking System } \\
\text { based on Face } \\
\text { Recognition" } \\
\text { Khem Puthea, } \\
\text { Rudy Hartanto } \\
\text { and Risanuri } \\
\text { Hidayat. }\end{array}$ & $\begin{array}{l}\text { The complete } \\
\text { system is } \\
\text { implemented } \\
\text { in MATLAB } \\
{[14]}\end{array}$ & $\begin{array}{l}\text { User-friendly } \\
\text { application on } \\
\text { face recognitions } \\
\text { created. }\end{array}$ & $\begin{array}{l}\text { Similar } \\
\text { techniques are } \\
\text { used. }\end{array}$ \\
\hline
\end{tabular}

\section{METHODOLOGY}

In order to mark attendance, we follow a series of steps which includes enrolment, face detection, face recognition, and then marking the attendance in a database. Unlike Eigenfaces and Fisherfaces, where in most modern face verification systems, training and enrolment are two different steps. Training is performed on millions of images. On the other hand, enrolment is performed using a small set of images. In case of Dlib, enrolling a person is simply passing a few images of the person through the network to obtain 128dimensional feature descriptors corresponding to each image. In other words, we convert each image to a feature in a high-dimensional space. In this high dimensional space, features belonging to the same person will be close to each other and far away for different persons.

\section{A. Traditional Image Classification Pipeline Versus Dlib's Face Recognition Model}

In a traditional image classification pipeline, we convert the image into a feature vector (or equivalently a point) in higher dimensional space. 
This was done by calculating the feature descriptor (e.g. HOG) for an image patch. Once the image is represented as a point in higher dimensional space, we then use a learning algorithm like SVM to partition the space using hyperplanes that separated points representing different classes.

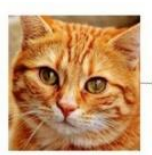

Input image
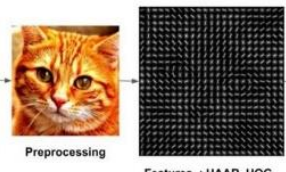

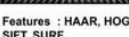

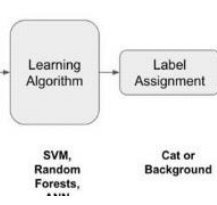

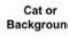

Figure 3: Traditional image classification pipeline.

Even though on the surface Deep Learning looks very different from the above model, there are conceptual similarities.

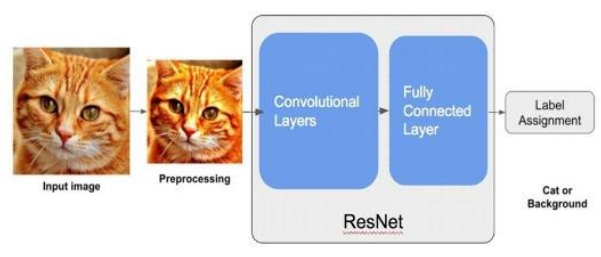

Figure 4: Dlib's Face Recognition module

Figure 4 reveals the Dlib's Face Recognition module is based on an CNN architecture called ResNet. ResNet contains a bank of Convolutional Layers followed by one Fully Connected Layer. As most CNN architectures, ResNet contains a bank of Convolutional (Conv) Layers followed by a Fully Connected (FC) Layer. The bank of convolutional layers produces a feature vector in higher dimensional space just like the HOG descriptor.

The most important differences between bank of convolutional layer and HOG descriptor are:

1. HOG is a fixed descriptor. There is an exact recipe for calculating the descriptor. On the other hand, a bank of conv layers contains many convolution filters. These filters are learned from the data. So unlike HOG, they adapt based on the problem at hand.

2. The FC layer does the same job as the SVM classifier in traditional approaches. It classifies the feature vector. In fact, sometimes the final FC layer is replaced by an SVM. Usually, when we want to use the word "distance" between two points we are talking about the Euclidean distance between them.

For example, the distance between $3 \mathrm{D}$ points $(1,0,1)$ and $(1,3,5)$ is

$\sqrt{\left.(1-1)^{2}+(3-0)^{2}+(5-1)^{2}\right)}=5$

In general, if we have an $\mathrm{n}$ dimensional vectors $\mathrm{x}$ and $y$ the L2 distance (also called the Euclidean distance) is given by

$d_{L 2}=\|x-y\|=\left[(x-y)^{T}(x-y)\right]^{\frac{1}{2}}=\left(\sum_{i=1}^{n}\left(x_{i}-y_{i}\right)^{2}\right)^{\frac{1}{2}}$
However, in mathematics a distance (also known as a metric) has a much broader definition. For example, a different kind of distance is called the L1 distance. It is the sum of absolute values of elements of the two vectors.

$$
d_{L 1}=\sum_{i=1}^{n}\left|x_{i}-y_{i}\right|
$$

The following rules define when a function involving two vectors can be called a metric. A mapping $d(x, y)$ is called a metric if

1. The distance between any two points is greater than or equal to zero $\mathrm{d}(\mathrm{x}, \mathrm{y}) \geq 0$

2. A point has zero distance from itself i.e., $d(x, x)=0$

3. The distance from $x$ to $y$ i.e., the same as the distance from $y$ to $x$ i.e., $d(x, y)=d(y, x)$

4. Triangle inequality: For any three points $x, y$ and $z$ the following inequality holds true. i.e.,

$\mathrm{d}(\mathrm{x}, \mathrm{y})+\mathrm{d}(\mathrm{y}, \mathrm{z}) \geq \mathrm{d}(\mathrm{z}, \mathrm{x})$

\section{1) Deep Metric Learning}

Any image can be vectorized by simply storing all the pixel values in a tall vector. This vector represents a point in higher dimensional space. However, this space is not very good for measuring distances. In a face recognition application, the points representing two different images of the same person may be very far away and the points representing images of two different people may be close by.

Deep Metric Learning is a class of techniques that uses Deep Learning to learn a lower dimensional effective metric space where images are represented by points such that images of the same class are clustered together, and images of different class are far apart. Instead of directly reducing the dimension of the pixel space, the convolution layers first calculate the meaningful features which are then implicitly used to create the metric space. Turns out we can use the same CNN architecture we use for image classification for deep metric learning.

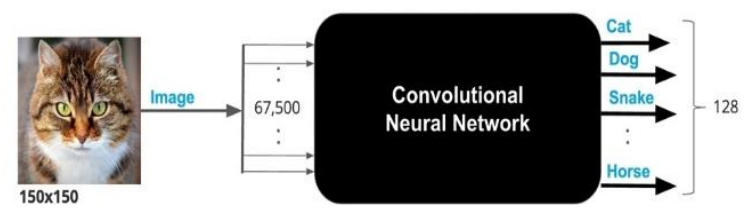

Figure 5: CNN for clarification task

In Figure 5 see a CNN that is trained to take as input a $150 \times 150$ colour image (which is the same as a vector of size $150 \times 150 \times 3=67,500$ ) and output the probability that it belongs to one of the 128 different animal classes. In Deep Metric Learning, the architecture remains the same, but the loss function is changed. 


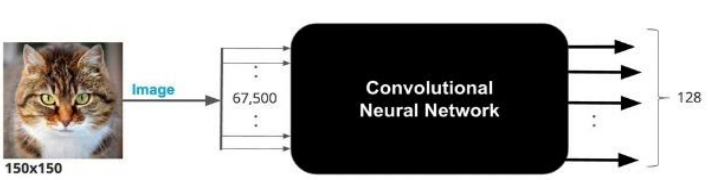

Figure 6: CNN for metric learning

Figure 6 reveals in Deep Metric Learning, the architecture remains the same as for $\mathrm{CNN}$ classification task, but the loss function is changed.

In other words, you input an image and the output is a point in 128-dimensional space. If you want to find how closely related two images are, you can simply find the pass both images through the $\mathrm{CNN}$ and obtain the two points in this 128-dimensional space. You can compare the two points using simple L2 (Euclidean) distance between them.

\section{2) Metric Loss}

Millions of images are typically used to train a production ready CNN. Obviously, these millions of images cannot be simultaneously used to update the knobs of the CNN. Training is done iteratively using one small batch of images at a time. This small batch is called a mini batch. As mentioned in the previous section, we need to define a new loss function so that the CNN output is a point in this 128-dimensional space. The loss function is defined over all pairs of images in a mini batch.

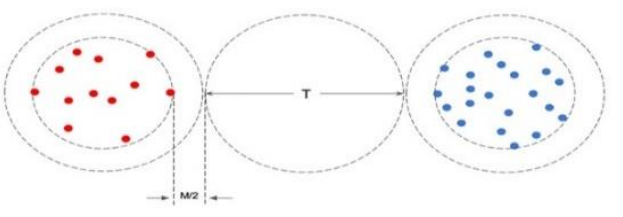

Figure 7: Metric loss defined by Dlib's Face Recogniser

For simplicity, the concept is shown in 2D. The loss is defined in terms of two parameters: 1) Threshold (T) and 2) Margin. The blue and the red dots present images of two different classes. For the metric loss to be 0 , the maximum distance between any two points of the same class should be (T - M) and the minimum distance between any two points of different classes should be $(\mathrm{T}+\mathrm{M})$ Let $\mathrm{p} 1$ and $\mathrm{p} 2$ represent the points corresponding to images 11 and 12 in the 128 dimensional space. If the images belong to the same class, the loss is given by $\max (0,\|\mathrm{p} 1-\mathrm{p} 2\|-\mathrm{T}+\mathrm{M})$

On the other hand, if 11 and 12 have two different class labels then their contribution to the loss function is: $\max (0, \mathrm{~T}-\|\mathrm{p} 1-\mathrm{p} 2\|+\mathrm{M}$

Figure 7 shows how this loss function prefers embedding where images of the same class are clustered together, and images of different classes are separated by a large margin.

\section{3) Hard Negative Mining}

In a mini batch, there are many non-matching pairs (images from different classes) than matching pairs (images from the same class). It is important to take this imbalance into account while calculating the metric loss function. If there are $\mathrm{N}$ matching pairs that share the same class in a mini batch, then the algorithm includes ONLY the $\mathrm{N}$ worst non-matching pairs in the loss computation. In other words, performs hard negative mining on the mini batch by picks the worst non-matching pairs.

\section{B. Enrolment}

For enrolment we define smaller ResNet neural network. Training was also done using this network. A Persons' images we are going to enrol are structured in following way: We will be having subfolders, each subfolder has images of one person. We will store this mapping of images and their corresponding labels to use it later in testing. Then we process enrolment images one by one, convert each image from BGR to RGB format, because Dlib uses RGB as default format. Then convert OpenCV BGR image to Dlib's cv_image and then Dlib's cv_image to Dlib's matrix format since Dlib's cv_image format is not recognized by neural network module. Detect faces in the image. For each face we detect facial landmarks and get a normalized and warped patch of detected face. Compute face descriptor using facial landmarks. This is a 128dimensional vector which represents a face. Then save labels and names to disk and face descriptors and corresponding labels to disk.

\section{Face Detection And Recognition}

Given a new image of a person, we can verify if it is the same person by checking the distance between the enrolled faces and the new face in the 128-dimensional space. Read name-labels mapping and descriptors from disk. Then read the query image that is an image of classroom with multiple students and convert it from BGR to RGB format. Because Dlib uses RGB as default format. Then convert OpenCV RGB image to Dlib's cv_image, and then Dlib's cv_imageto Dlib's matrix format. Dlib's cv_image format is not recognized by neural network module. Detect faces in query image. For each face detect facial landmarks. Get a warped and patch of 150x150 for each face. Now compute face descriptor for each face. Now we calculate Euclidean distance between face descriptors in query images versus face descriptors of enrolled images. Find the enrolled face for which distance is minimum. Dlib specifies that in general, if two face descriptor vectors have a Euclidean distance between them less than 0.6 then they are from the same person, otherwise they are from different people. This threshold will vary depending upon number of images enrolled and various variations (illumination, camera quality) between enrolled images and query image. We are using a threshold of 0.5 . If minimum distance is less than threshold, find the name of person from index, else the person in query image is unknown. 


\section{Attendance Marking}

For each face detected and matched with enrolled face, the attendance is marked for the corresponding USN in the database. The name of student along with day and time of attendance is also be stored in the database.

\section{CONCLUSION}

The above method provides the best outcome will be achieved. This is achieved using OpenCV for frame extraction and dlib for face recognition. This method will have higher accuracy in recognition of multiple faces from a single frame with lower response time.

\section{REFERENCES}

[1] Xin Geng, Zhi-Hua Zhou, \& Smith-Miles, K. (2008). Individual Stable Space: An Approach to Face Recognition Under Uncontrolled Conditions. IEEE Transactions on Neural Networks.

[2] Winarno, Wiwien Hadikurniawati, Imam Husni Al Amin, Muji Sukur, "Anti-Cheating

[3] Presence System Based on 3WPCA Dual Vision Face Recognition, Faculty of Information Technology Universitas Stikubank Semarang Indonesia.

[4] Prototype model for an Intelligent Attendance System based on facial Identification by Raj Malik, Praveen Kumar, Amit Verma, Seema Rawat, Amity University Uttar Pradesh.

[5] Convolutional Neural Network Approach for Vision Based Student Recognition System, Nusrat Mubin Ara1, Dept. of CSE, SUST, Sylhet, Bangladesh

[6] NFC Based Mobile Attendance System with Facial Authorization on Raspberry Pi and Cloud Server Siti Ummi Masruroh Andrew Fiade Imelda Ristanti Julia.

[7] Face recognition-based Attendance System using Machine Learning Algorithms, Radhika C. Damale, Department of Electronics and Telecommunications, Cummins College of engineering for Women, Pune, Maharashtra, India.

[8] Class Attendance system based on Face Recognition" Priyanka Wagh.

[9] Design of Classroom Attendance System Based on Face Recognition,WenxianZeng.

[10] Automated Attendance System Using Face Recognition, Akshara Jadhav, Akshay Jadhav Tushar Ladhe, Krishna Yeolekar.

[11] An Attendance Marking Systembased on Face Recognition" written by Khem Puthea, Rudy Hartanto and Risanuri Hidayat.

[12] Class Attendance Management System Using Face Recognition ,Omar Abdul Rhman Salim Department of Electrical and Computer Engineering, Faculty of Engineering International Islamic University Malaysia, Kuala Lumpur,Malaysia o.salem92@gmail.com

[13] Face Recognition Based Attendance System Nandhini R, Duraimurugan N.

[14] Student Attendance System in Classroom Using Face Recognition Technique, Samuel LukasAditya Rama Mitra, Ririn Ikana Desanti, Dion Krisnadi, Informatics Department,Computer System Department, Information System Department Universitas Pelita Harapan Karawaci, Indonesia.

[15] [14] Attendance System based on Face Recognition Venkata Kalyan Polamarasetty, Muralidhar Reddy Reddem, Dheeraj Ravi, Mahith Sai Madala. 